\title{
Cancer diagnosis in primary care
}

\author{
William Hamilton
}

\begin{abstract}
Around a quarter of those in the developed world die of cancer. Most cancers present to primary care with symptoms, even when there is a screening test for the particular cancer. However, the symptoms of cancer are also symptoms of benign disease, and the GP has to judge whether cancer is a possible explanation. Very little research examined this process until relatively recently. This review paper examines the process of primary care diagnosis, especially the selection of patients for rapid investigation. It concentrates on the four commonest UK cancers: breast, lung, colon, and prostate as these have been the subject of most recent studies.

Keywords

cancer; diagnosis; primary health care.
\end{abstract}

\section{INTRODUCTION}

Around a quarter of those who live in the developed world will die of cancer. This figure is rising, as competing causes of death diminish in importance. Most of those with cancer present with symptoms, and most of these presentations are to primary care. ${ }^{1}$ Although dealing with new cancer diagnoses is relatively rare, (a full-time UK GP will have a new cancer diagnosed in one of his or her patients each month) dealing with the possibility of cancer is an everyday occurrence. Thus GPs rapidly become highly experienced in diagnosing what is not cancer, and slowly become experienced in diagnosing what is cancer. What is irksome to GPs is that they are judged almost entirely on their diagnostic performance in those patients who transpire to have cancer, while given little or no credit for their performance in those who do not.

In theory, cancer diagnosis should have become easier in the last decade. In the UK, rapid investigation clinics, which offer specialist investigation within 2 weeks, are well established. Selection of patients is guided by national recommendations, ${ }^{2}$ although these are frequently ignored. ${ }^{3}$ Access to primary care imaging has improved, both in terms of availability and timeliness. Even so, the UK's record in cancer mortality remains poor compared with other countries, ${ }^{4}$ and it is argued that all these new facilities have made little or no difference. ${ }^{5}$ The current estimate is that $7500-10000$ lives are lost each year in the UK from late diagnosis. ${ }^{6}$ However, this headline figure conflates several separate aspects of diagnosis, illustrated in Figure 1.

Almost all research has examined the whole interval A-E, with only a few studies teasing out where delays are occurring, and where there is the most potential for improvement. ${ }^{7-10}$ For the rest of this review, I concentrate upon activity between $\mathrm{B}$ and $D$, so from first presentation to referral for definitive investigation and take most of my examples from the four commonest cancers, breast, prostate, lung, and colorectal.

In retrospect, it is usually easy for the patient to pinpoint when their symptoms began and when they were first reported to primary care. ${ }^{11-12}$ At the time, however, it may be far from obvious to the clinician that the symptoms represent a malignancy. There is reasonable evidence that patients can 


\section{How this fits in}

There has been an upsurge in cancer diagnosis research in recent years, especially studies based in primary care. Selection of patients for investigation has always been complex. This review summarises recent advances, particularly those supporting a 'risk-based' approach to referral. guidance, while simultaneously stating that referral guidance has a very poor ability to identify cancer. ${ }^{3}$ This inconsistency leaves the GP in the middle, balancing patient expectations against the need to use resources appropriately. For the rest of this review, I will summarise recent advances in primary cancer diagnosis, to try and help in this balancing act.

identify which symptoms matter. Most symptoms that could represent cancer are not reported to primary care, as the patient makes the - usually correct - assessment that there is a simple explanation for their symptom, and one which does not require medical attention. Thus, only $41 \%$ of patients with rectal bleeding report it to their doctor. ${ }^{13}$ There are two selection processes when a patient has a symptom: the first being whether to seek medical attention, and the second being when the GP decides whether to refer or not. Across both these selection processes the risk of cancer is higher in those who have been selected (understandably - as the selection process works, albeit imperfectly). Interestingly, the relative increase in cancer risk over the first selection (deciding whether to consult) is greater than the second selection (the GP deciding whether to refer). ${ }^{14}$ As an example, the positive predictive value (or risk) of colorectal cancer with a single episode of rectal bleeding experienced in the community is around $0.1 \% .^{15}$ Once the bleeding is reported to primary care, the risk of cancer is approximately $2-3 \% .{ }^{16-17}$ In patients referred to secondary care, the risk is $5-7 \% .^{18}$

This gradient of risk is important. Much discussion centres on the 'gatekeeper' role of primary care, whereby GPs select patients for investigation or referral. It is possible that the UK's relatively poor cancer performance may result from GPs having too high a threshold for selecting patients for investigation. However, specialist clinics are quick to criticise GPs for their failure to comply with referral

\section{Breast cancer}

For such an important and emotive cancer, there is remarkably little primary care evidence to guide GPs in their referrals of women with breast symptoms. Hundreds of studies have examined screening or genetics, yet bizarrely more primary care research addresses GP compliance with referral guidance than research which provides evidence to formulate this guidance. It is not as if most breast cancers are identified as a result of screening; on the contrary, three-quarters are diagnosed following presentation with symptoms. ${ }^{1}$

Breast symptoms account for almost 3\% of female consultations in primary care. The commonest reported are breast pain and masses, with such complaints commonest in the $25-44$ age group. ${ }^{19}$ Complaints of nipple discharge or changes in the skin of the breast are much rarer..$^{20} \mathrm{~A}$ breast lump has a likelihood ratio of 15 , whereas for a nipple complaint the figure is 3.1 and for breast pain 1.8. ${ }^{19}$ The two common metrics used in diagnosis are described in Box 1. Overall, $8 \%$ of women with a breast lump reported to primary care are subsequently diagnosed with cancer. As would be expected, this figure is highly dependent upon age; the incidence of breast cancer being 15 times higher in women aged 45-64 years than in those aged below 25 years. ${ }^{19}$ Thus in those below 25 years, a breast lump has an approximate risk of cancer of $0.5 \%$ - assuming the likelihood ratios are similar across all ages, which is generally the case in cancer diagnostic studies. ${ }^{17}$ Less than $2 \%$ of women with nipple complaints have cancer. The
Figure 1. Intervals used in the literature of diagnostic delays.

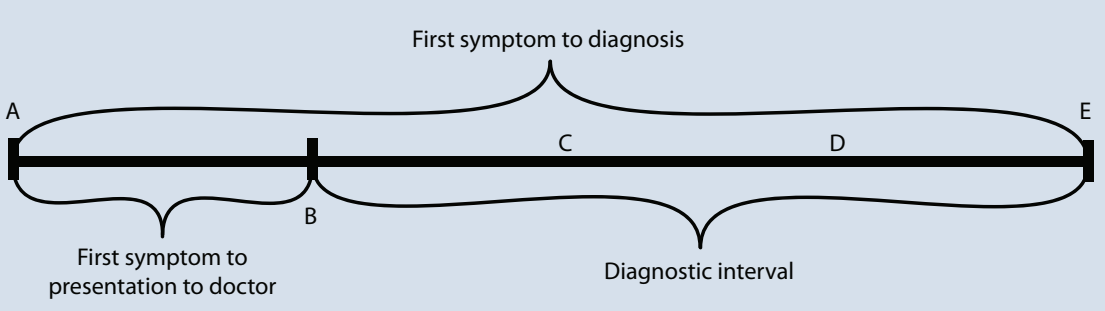

A denotes the timing of the first experience of a symptom; $B$ the first attendance for medical care (usually in primary care'), $C$ the clinician first recognises cancer as a possibility, D definitive investigation is begun (which often coincides with $C$ ) and $E$ the start of treatment. This timeline begins with symptoms, although many cancers have a pre-symptomatic stage before A. 
percentage for breast pain is even lower at $0.9 \%$. Such figures, which were published after NICE guidance, support the concept of an age threshold for urgent referral of breast lumps. Indeed, it is debatable whether nipple bleeding (which usually represents duct ectasia) warrants such prominence in referral guidance, as such a small proportion of women with this symptom have an underlying cancer. Similarly, breast pain is genuinely a very low-risk symptom.

In younger women, GPs have to rely upon their clinical skills to identify the suspicious lump, although many women will end up being referred as the only method of providing adequate reassurance. Younger women are particularly at risk of inflammatory breast cancer (which accounts for around $5 \%$ of all breast cancers). As the name suggests, swelling, redness, and warmth of the breast are the main features. 'Peau d'orange' changes in the skin are common. ${ }^{21}$ These appear quite rapidly, making differentiation from infection difficult. The underlying pathology is blockage of the local lymph vessels by cancer cells, so the prognosis of this form of breast cancer is poorer.

\section{Lung cancer}

Lung cancer diagnosis attracts much nihilism, even fatalism. The prognosis is generally poor, and many people question how much is to be gained from early diagnosis. ${ }^{22}$ Patients may share this nihilism, in some from a feeling of guilt at a self-inflicted illness. ${ }^{23}$ This can delay presentation of symptoms. By the time symptoms have been reported the cancer has generally spread, making curative treatment impossible. Furthermore, the symptoms of lung cancer are usually non-specific, so even if the patient presents early, it is often difficult to identify that cancer is the cause. ${ }^{24}$ These factors mean that only $20 \%$ of UK patients with lung cancer are eligible for surgical resection; ${ }^{25}$ only $17 \%$ actually undergo surgery, ${ }^{26}$ and around half of those survive 5 years.

As yet, screening has little to offer. ${ }^{27}$ Conventional sputum cytology does not work, as its sensitivity is too low; nor does plain chest X-ray screening. Annual cytology, augmented by computer-assisted image analysis, in smokers with chronic obstructive pulmonary disease is currently being studied in the UK Lung-SEARCH trial. Two large screening trials of spiral CT are in progress, with the US trial due to report its final results in 2010. The European trial reports later that decade. However, the 3-year results of a smaller recent Italian trial were disappointing. ${ }^{28-29}$ Spiral CT can detect nodules as small as $0.5 \mathrm{~cm}$, compared with $3 \mathrm{~cm}$ for plain radiology. ${ }^{25}$ However, the problem with being able to

\section{Box 1. Metrics used in diagnostic research}

Likelihood ratio. This is the chance of a patient with cancer having a symptom of the cancer, divided by the chance of a patient without cancer having the same symptom. For example, in lung cancer, $20 \%$ of cases report haemoptysis to their doctor, whereas $1.5 \%$ of 'healthy' people do so. In this example, the positive likelihood ratio is 20/1.5 $=13$.

Positive predictive value. This is the chance of a patient having the disease of interest when they have reported the symptom. For the same example, a person reporting haemoptysis to their GP has a $2.4 \%$ chance of the symptom being due to an underlying lung cancer.

There is a relationship between these two metrics. The positive predictive value for the symptom is the incidence of the relevant cancer multiplied by the likelihood ratio for the symptom. Strictly, it is slightly more complicated than that, in that the positive predictive value and the incidence have to be expressed as odds for the calculations, and then converted back to percentages. Furthermore, you have to decide the period that a symptom is deemed to be relevant (a cough more than a year before lung cancer is diagnosed is probably not due to the cancer). Even so, the essential point is that the larger the likelihood ratio, the higher the positive predictive value. Similarly, the lower the incidence (that is, the rarer the cancer), the lower the positive predictive value.

identify such small nodules is the very high falsepositive rate: at $21 \%$ after one scan and $33 \%$ after two. The false-positive rate may be lower in the highly targeted population of the Lung-SEARCH trial, as the incidence will be higher in this high-risk population.

As there is generally a long period of symptoms before presentation to a doctor, ${ }^{11,30}$ efforts have been made to increase symptom awareness, and thus earlier presentation. Such initiatives are difficult to research, but audits suggest a moderate effect in terms of earlier staging, with a concomitant increase in resectability. A campaign in Doncaster, UK, encouraged patients with a cough to report it to their GP. This was coupled with a liberalisation of criteria for requesting a chest X-ray. The percentage of cancers identified in stages I or II increased from 11 to $19 \%$ after this campaign - a significant rise $(P=0.02) .{ }^{31}$ In my view, it is much more likely that progress will be made from interventions such as this, than through screening - although most lung cancers will remain incurable.

In one aspect, lung cancer is easier than most other cancers, as the main test (a chest X-ray) is easily available, reasonably cheap, and quite accurate. Roughly one chest X-ray in five requested by primary care is for investigation of possible lung cancer. ${ }^{32}$ Negative $X$-rays occur in a quarter of cancers, ${ }^{33}$ with some lesions being missed by the radiologist, and others being not visible. ${ }^{34}$ Even so, a quarter is a relatively low figure for a primary care cancer test (for example, the haemoglobin is over $12.9 \mathrm{~g} / \mathrm{dl}$ in about half of those 
with colorectal cancer). ${ }^{35}$ There are relatively few false-positive chest $X$-rays (reflecting the $3 \mathrm{~cm}$ minimum size of nodule that can be reliably identified), although many chest $\mathrm{X}$-rays show an indistinct abnormality requiring a repeat test. Therefore, it is reasonable to have a low threshold for requesting a chest $\mathrm{X}$-ray in a patient with symptoms that could represent lung cancer. If the threshold for ordering a chest X-ray were to be liberalised, the yield of additional cancers would still be small. Unpublished estimates from a UK primary care cohort examined a possible policy of X-raying all current smokers over the age of 60 years presenting to primary care with a cough. ${ }^{36-37}$ Such a policy would expedite the diagnosis in a quarter of those with lung cancer by a median of 9 months. This could have a mortality benefit. However, the 'cost' would be borne by those without cancer - the number needed to Xray to find one of these 9-month expedited cancers is 47 . This may be a good use of resources, when compared with the cost and false-positive rate of screening.

For the patient who has reported symptoms to primary care, there is recent evidence as a basis for investigation and/or referral decisions. The obvious symptom is haemoptysis, which carries a risk of 2.4-7.5\% depending upon age and sex. ${ }^{36,38}$ Such figures clearly warrant a chest X-ray. However, haemoptysis is relatively uncommon in lung cancer, being reported to primary care by only $40 \%$ of patients. ${ }^{36}$ All other symptoms - cough, dyspnoea, loss of appetite, loss of weight, fatigue, and chest pain - are more common in cancer than haemoptysis. However, they are even more common in benign conditions, so have positive predictive values of less than $1 \%$ for lung cancer. Positive predictive values are higher in smokers or those over 70 years, ranging from $1-2 \%{ }^{39}$ Arguably these latter two groups warrant a chest X-ray at a risk of this magnitude. Such a low yield is justifiable, given the relatively low cost and ease of access to chest X-rays. For other cancers, where the test is not so simple, there is still considerable debate as to what the threshold value of risk warranting investigation should be: patients, clinicians and commissioners of care having different views. Despite the non-specific nature of symptoms in the majority of those with lung cancer, the median GP delay (B-D in the figure) in the UK is 51 days, ${ }^{37}$ although it is 26 days in Sweden. ${ }^{30}$ As is the case with most cancers, the majority of patients presenting with symptoms are identified and investigated relatively early, with a minority (albeit a very important one) experiencing significant delays.

\section{Colorectal cancer}

This cancer has received the most attention in recent years with one published systematic review (incorporating secondary care as well as primary care data), and two primary care ones reporting shortly. ${ }^{40}$ Several recent studies have examined rectal bleeding. ${ }^{16-17,38,41-45}$ No study has reported a risk for rectal bleeding below $2 \%$, except in those under 60 years of age. ${ }^{17}$ Current UK referral guidance requires 6 weeks of bleeding or bleeding accompanied by diarrhoea for an urgent referral. ${ }^{2}$ Even ignoring the illogicality of waiting 6 weeks so one can then have a 2 -week referral, I believe that the risk from rectal bleeding per se in the over-60s warrants investigation, whatever accompanying symptoms there are.

If guidance on rectal bleeding is debatable, it is simply wrong with respect to iron-deficiency anaemia. The threshold value of haemoglobin plus iron-deficiency at which rapid investigation haemoglobin for colorectal cancer is recommended is $10 \mathrm{~g} / \mathrm{dl}$ in women and $11 \mathrm{~g} / \mathrm{dl}$ in men. These equate to risks of colorectal cancer of $13.3 \%$ and $7.7 \%$ respectively. ${ }^{35}$ Clearly, patients with milder levels of anaemia warrant investigation for possible colorectal cancer, especially as this is the symptom of colorectal cancer associated with a worse mortality. ${ }^{46}$

Referral decisions become much more difficult when considering the low-risk-but-not-no-risk symptoms, such as abdominal pain, constipation, or diarrhoea. These have positive predictive values of $1 \%$ or less. ${ }^{16-17}$ For lung cancer, it is easy to advocate a liberalisation of investigation of lowrisk-but-not-no-risk respiratory symptoms, as the chest $\mathrm{X}$-ray is easily available, of minimal risk and discomfort, and with reasonable sensitivity and specificity. However, colonoscopy is more costly and uncomfortable, although it also has good performance characteristics. ${ }^{47}$ One hope was that CT colonography could replace colonoscopy however, it misses one in 10 of lesions larger than $1 \mathrm{~cm}$ - which are not missed by colonoscopy. ${ }^{48}$ Also possible for the future are various biomarkers, ${ }^{49}$ including a small protein, matrix metalloproteinase-9. Serum levels of this protein correlate with the presence of colorectal cancer. ${ }^{50}$ Similarly, free DNA can be identified in the rectum of some patients with colorectal cancer..$^{51}$ Like the biomarkers, much more research is required before this can be used in primary care. Much simpler than these methods are scoring systems for use in those with a low-risk-but-not-no-risk symptom. Two have been advocated: the CAPER score and the SELVA score. ${ }^{18,52}$ Both have similar problems - achieving high sensitivity (that is, ensuring most of those with 
cancer are identified) requires a sacrifice of specificity, with many patients undergoing negative investigations, despite being labelled by the scores as 'higher risk'. ${ }^{53}$

Until one of these initiatives is shown to be successful, GPs have to rely on softer clues to identify colorectal cancer in patients with a low-risk symptom. This can be done, and done well. An example: the expression 'change in bowel habit' strictly just means constipation or diarrhoea. Yet the expression has connotations beyond its dictionary definition. In the UK, when a GP uses that expression, it means 'constipation or diarrhoea, and there is a reasonable chance this is due to a colorectal cancer.' Very few patients have change in bowel habit documented in their records, yet are not investigated for possible cancer, whereas the majority of patients with, for example, diarrhoea documented in their records go without investigation. And GPs get this right: the positive predictive value of 'change in bowel habit' in a 80 year old man is $4 \%$ compared with $1.2 \%$ and $0.7 \%$ for the same age of man with diarrhoea or constipation. ${ }^{17}$ Some additional features must allow GPs to identify these patients - who are at least three times more likely to harbour cancer. What these features are and how they are detected by the GP is not known - that they exist seems certain. It is unlikely that GPs consciously act in a Bayesian fashion in calculating a prior probability of cancer and adjusting it for these subtle clues. Subconsciously, that may be exactly what they are doing.

\section{Prostate cancer}

As with lung cancer, there is some nihilism about the usefulness of early diagnosis of prostate cancer, although from a different direction. Put crudely, the argument goes that early diagnosis of lung cancer is no use as the patient will die anyway; early diagnosis of prostate cancer is no use as the patient will live anyway. Much of the prostate cancer nihilism stems from screening trials. Recently published studies of screening for prostate cancer show very little evidence of mortality benefit. ${ }^{54-55}$ Treatment of small cancers does yield a small mortality gain, but at a cost of possible complications such as incontinence. ${ }^{56}$ However, treatment of larger tumours, and of disease that has spread is much less controversial. ${ }^{57}$ Larger tumours are also those likely to give rise to symptoms. This simplifies the clinical problem for primary care. Given that there is benefit for treatment of symptomatic prostate cancer, it is appropriate to search for prostate cancer when a man presents with lower urinary tract symptoms. However, lower urinary tract symptoms essentially mean the prostate gland is enlarged, but do not tell whether the enlargement is benign or malignant. ${ }^{58-59}$ Most of the common lower urinary tract symptoms have positive predictive values for prostate cancer of around $3 \% .^{58}$ Additionally, impotence can be a first (and early) symptom of cancer, again with a risk of approximately $3 \%{ }^{58}$ Thus in men with lower urinary tract symptoms, clinical management should include rectal examination and measurement of the prostate specific antigen (PSA). ${ }^{60}$ Such use of the PSA is entirely different from its use as a screening tool. Although a small number of men can have prostate cancer with a PSA as low as $1 \mathrm{ng} / \mathrm{ml},{ }^{61}$ almost all such cancers have a good prognosis, and missing them is of limited importance. ${ }^{62}$ Urologists sometimes use the velocity of PSA rise, or the level of free PSA in selection of patients for biopsy. ${ }^{63}$ There is no published evidence to support such measurements in primary care.

\section{Ovarian cancer}

Quite a few primary care based studies have recently illuminated this difficult cancer. It is now clear that the term 'silent killer' is wrong. All primary care studies show that symptoms are common, and are generally reported to GPs. ${ }^{64-69}$ It is actually a 'noisy killer.' The problem is that the noise is nonspecific, with symptoms such as fatigue, abdominal pain and urinary frequency being common initially. Abdominal distension has a relatively high risk of cancer, with a positive predictive value of $2.5 \%$, even though it is omitted from current guidance. ${ }^{69}$ One problem seems to be that GPs understandably - either don't think of ovarian cancer with such symptoms, or consider it so unlikely as not to warrant examination or investigation. Thus, ovarian cancer is often seen as one GPs frequently miss. ${ }^{12}$ The truth is that GPs are probably no worse (or better) at diagnosing ovarian cancer than they are at any internal cancer. ${ }^{70}$ It is possible to measure serum CA125 in primary care; this has reasonable specificity, but the sensitivity is unknown, so it is of debatable value..$^{71-72} \mathrm{~A}$ better test is ultrasound, ideally trans-vaginally. This has been extensively tested in screening studies, with good performance characteristics, although - as always - it is risky to extrapolate from the screened population to the symptomatic population. ${ }^{73} \mathrm{~A}$ large UK screening trial has completed recruitment, but the main results will not be available until after 2014, when mortality followup ceases. ${ }^{73}$

\section{Other cancers}

Few other cancers have been researched in primary 
care - largely because of their relative rarity. Thus, referral decisions have to be based on the GP's experience - allied to intuition - with intuition being probably the more important. What few studies have been done largely support current standard clinical practice. Thus, the risk of oesophageal cancer with dysphagia is $5.7 \%$ in men and $2.4 \%$ in women and the risk of urinary tract malignancy with haematuria $7.4 \%$ and $3.4 \%$ respectively, suggesting investigation is warranted..$^{38}$ Similarly, GPs appear to be good at identifying which patients with enlarged cervical lymph nodes have cancer, and equally importantly, which do not. $^{74-75}$ Overall, less than $2 \%$ of cervical lymphadenopathy presented to primary care is malignant. ${ }^{76}$ The risk of a brain tumour with headache is 1 in 1000, supporting the view that scanning patients with typical headache is not required. ${ }^{77}$

\section{Summary}

Primary care is the main setting where cancer is diagnosed, or at least suspected. It is also the setting in which most pre-symptomatic risk assessment takes place - including compilation of a family history, and attention to modifiable risk factors, such as smoking or obesity. ${ }^{78}$ Hardly a day in clinical practice passes without cancer being raised as a possibility. The evidence base behind selection of patients for referral - and equally importantly - behind reassuring and not investigating continues to grow. At times, the evidence runs counter to current guidelines: if so most GPs trust their clinical acumen, and are generally right to do so. ${ }^{79}$ Most patients with cancer receive good service from primary care. Further research will need to explore how the minority who currently experience delays in diagnosis can be identified earlier.

\section{Linked commentary}

This article has an associated commentary: Neal RD. Cancer diagnosis - the role of urgent referral guidelines. $\mathrm{Br}$ J Gen Pract 2010; DOI: 10.3399/bjgp10X483427.

\section{Funding}

No specific funding. William Hamilton holds a NIHR postdoctoral fellowship. The views expressed in this publication are his and not necessarily those of the Department of Health

\section{Acknowledgements}

Peter Wood critiqued an earlier draft offering several helpful suggestions.

\section{Competing interests}

William Hamilton has received funding for several of his studies, including NIHR School of Primary Care Research, the Department of Health, CRUK, and a commercial company, Colonix Ltd., who manufacture a device that captures rectal DNA. None had any input into this review

\section{Discuss this article}

Contribute and read comments about this article on the Discussion Forum: http://www.rcgp.org.uk/bjgp-discuss

\section{REFERENCES}

1. Hamilton W. Five misconceptions in cancer diagnosis. Br J Gen Pract 2009; 59: 441-447.

2. NICE. Referral Guidelines for suspected cancer. London: NICE, 2005. http://www.nice.org.uk:80/nicemedia/pdf/cg027niceguideline.pdf (accessed 18 Dec 2009).

3. Ballal M, Selvachandran S, Maw A. Use of patient consultation questionnaire and weighted numerical scoring system for the prediction of colorectal cancer and other colorectal pathology in symptomatic patients. A prospective cohort validation study of a Welsh population. Colorectal Disease 2009; 9999(999A).

4. Gondos A, Bray F, Brewster DH, et al. Recent trends in cancer survival across Europe between 2000 and 2004: A model-based period analysis from 12 cancer registries. Eur J Cancer 2008; 44: 1463-1475.

5. Rachet B, Maringe C, Nur U, et al. Population-based cancer survival trends in England and Wales up to 2007: an assessment of the NHS cancer plan for England. Lancet Oncol 2009; 10: 351-369.

6. Abdel-Rahman M, Stockton D, Rachet B, et al. What if cancer survival in Britain were the same as in Europe: how many deaths are avoidable? Br J Cancer 2009; 101(S2): S115-S24.

7. Richards MA, Sainsbury J, Ramirez A, et al. Influence of delay on survival in patients with breast cancer: a systematic review. Lancet 1999; 353: $2155-2162$

8. Rupassara KS, Ponnusamy S, Withanage N, Milewski PJ. A paradox explained? Patients with delayed diagnosis of symptomatic colorectal cancer have good prognosis. Colorectal Disease 2006; 8: 423-429.

9. Neal RD, Allgar VL, Ali N, et al. Stage, survival and delays in lung, colorectal, prostate and ovarian cancer: comparison between diagnostic routes. Br J Gen Pract 2007; 57: 212-219.

10. Kirwan JM, Tincello DG, Herod JJ, et al. Effect of delays in primary care referral on survival of women with epithelial ovarian cancer: retrospective audit. BMJ 2002; 324: 148-1451.

11. Corner J, Hopkinson J, Fitzsimmons D, et al. Is late diagnosis of lung cancer inevitable? Interview study of patients' recollections of symptoms before diagnosis. Thorax 2005; 60: 314-319.

12. Reeve G, Mackay-Thomas S. The invisible worm: ovarian cancer. 10.1136/bmj.b2072. BMJ 2009; 338: b2072

13. Crosland A, Jones R. Rectal bleeding: prevalence and consultation behaviour. BMJ 1995; 311: 486-488.

14. Hamilton W, Sharp D. Diagnosis of colorectal cancer in primary care: the evidence base for guidelines. Fam Pract 2004; 21: 99-106.

15. Fijten G, Muris J, Starmans R, et al. The incidence and outcome of rectal bleeding in general practice. Fam Pract 1993;10: 283-287.

16. Hamilton W, Round A, Sharp D, Peters T. Clinical features of colorectal cancer before diagnosis: a population-based case-control study. $\mathrm{Br} \mathrm{J}$ Cancer 2005; 93: 399-405.

17. Hamilton W, Lancashire R, Sharp D, et al. The risk of colorectal cancer with symptoms at different ages and between the sexes: a case-control study. BMC Medicine 2009; 7: 17.

18. Selvachandran S, Hodder R, Ballal M, et al. Prediction of colorectal cancer by a patient consultation questionnaire and scoring system: a prospective study. Lancet 2002; 360: 278-283.

19. Eberl MM, Phillips RL, Jr, Lamberts $\mathrm{H}$, et al. Characterizing breast symptoms in family practice. Ann Fam Med 2008; 6: 528-533.

20. Newton P, Hannay DR, Laver R. The presentation and management of female breast symptoms in general practice in Sheffield. 10.1093/fampra/16.4.360. Fam Pract 1999;16: 360-65.

21. Anderson WF, Schairer C, Chen BE, et al. Epidemiology of inflammatory breast cancer (IBC). Breast Disease 2005; 22: 9-23.

22. National Cancer Research Institute. Report of the NCRI strategic planning group on lung cancer. Lung cancer research in the UK 2006. London: NCRI, 2006.

http://www.parliament.uk/deposits/depositedpapers/2008/DEP2008 1838.pdf (accessed 22 Dec 2009)

23. Corner J, Hopkinson J, Roffe L. Experience of health changes and reasons for delay in seeking care: a UK study of the months prior to the diagnosis of lung cancer. Soc Sci Med 2006; 62:1381-1391.

24. Weller D, Campbell C. Early lung cancer detection: the role of primary care. Prim Care Resp J 2006; 15: 323-325.

25. Read C, Janes S, George J, Spiro S. Early lung cancer: screening and detection. Prim Care Resp J 2006; 15: 332-336.

26. Devbhandari MP, Yang SS, Quennell P, et al. Lung cancer resection rate in south Manchester: is it comparable to international standards? Results of a prospective tracking study. Interact CardioVasc Thorac Surg 2007; 6: 712-714. 
27. Silvestri GA, Alberg AJ, Ravenel J. The changing epidemiology of lung cancer with a focus on screening. BMJ 2009 (Aug 17); 339: b3053.

28. Infante M, Cavuto S, Lutman FR, et al. A randomized study of lung cancer screening with spiral computed tomography: three-year results from the DANTE trial. Am J Resp Crit Care Med 2009; 180: 445-453.

29. Bepler G. Are we coming full circle for lung cancer screening a second time? Am J Resp Crit Care Med 2009; 180: 384-385.

30. Lovgren M, Levealahti $H$, Tishelman C, et al. Time spans from first symptom to treatment in patients with lung cancer - the influence of symptoms and demographic characteristics. Acta Oncologica 2008; 47: 397-405.

31. NAEDI newsletter. Early Lung Cancer Intervention in Doncaster
(ELCiD). London: CRUK, 2009

http://info.cancerresearchuk.org/prod_consump/groups/cr_common/@

nre/@hea/documents/generalcontent/013777.pdf (accessed 22 Dec 2009).

32. Speets AM, van der Graaf Y, Hoes AW, et al. Chest radiography in general practice: indications, diagnostic yield and consequences for patient management. Br J Gen Pract 2006; 56: 574-578.

33. Stapley S, Sharp D, Hamilton W. Negative chest X-rays in primary care patients with lung cancer. Br J Gen Pract 2006; 56: 570-573.

34. White CS, Flukinger T, Jeudy J, Chen JJ. Use of a computer-aided detection system to detect missed lung cancer at chest radiography. Radiology 2009; 252: 273-281.

\section{COMMENTARY}

\section{Cancer diagnosis - the role of urgent referral guidelines}

This excellent review brings together evidence from primary care that will undoubtedly help clinicians in their quest for earlier cancer diagnoses for their patients. ${ }^{1}$ It is particularly welcome within the current context of the Department of Health's National Awareness and Early Diagnosis Initiative. ${ }^{2}$ Recent policy announcements have focused on easier and quicker access to investigations for GPs for suspected cancer symptoms, and the development and implementation of software for GPs to identify early cancer symptoms within 5 years. Such policies are likely to help save the estimated 5000-10 000 lives per year currently lost in the UK because of probable later diagnosis compared with some European countries. ${ }^{3-5}$ Data from primary care, such as presented in Hamilton's review, will directly inform the development of these policies.

One key policy question is whether urgent referral guidelines are the answer, whatever the quality and quantity of the evidence about the meaning of symptoms. There is a continuing lack of evidence that cancer diagnoses overall are made quicker, and with clinical benefits, through a fast-track system that prioritises some patients over others, based upon their symptoms. Many, quite possibly a majority, of cancers are diagnosed through a more atypical symptomatic presentation, often congruent with symptoms of benign pathology. This is a major challenge for secondary care and for commissioners. Paradoxically, guidelines may prioritise those with least to gain in terms of improved outcomes because they hasten diagnosis of very early tumours that may remain curable and treatable if diagnosed later. They also prioritise those with already established aggressive disease who are beyond curative treatment at the time of diagnosis, although good palliative care for these people may be hastened. ${ }^{6}$ All patients deserve the earliest diagnosis, whatever their symptoms. Guidelines do focus GPs' attention on potential alarm symptoms for cancer. ${ }^{7}$ However, there are also still significant concerns about the implementation and use of the urgent cancer referral guidelines in primary care, which is hugely different to their target driven implementation in secondary care.

In policy terms, there are two main conclusions to be drawn. Firstly, Hamilton is correct in stating that GPs may have too high a threshold for investigation. Gatekeeping has been the cornerstone of the UK NHS for decades. However, for suspected cancer, gatekeeping may be inhibiting early diagnosis and it may be that the balance between referral for opinion or investigation, and minimising costs and the risk of iatrogenic illness needs to be shifted. Watching and waiting is no longer acceptable for certain symptom complexes. Secondly, we need faster routes to diagnostic tests and/or specialist opinion (dependent upon the available diagnostic tests for different cancers) for all patients with a suspicious symptom, above a certain, and as yet undetermined, threshold. A stronger evidence base to enhance our understanding of the predictive value of symptoms will facilitate this.

\section{Richard D Neal,}

Senior Lecturer in General Practice, North Wales Clinical School, Department of Primary Care \& Public Health, School of Medicine, Cardiff University, Gwenfro Unit 5, Wrexham Technology Park, Wrexham, LL13 7YP. E-mail: nealrd@cf.ac.uk.

\section{Provenance}

Commissioned; not peer reviewed.

\section{REFERENCES}

1. Hamilton W. Cancer diagnosis in primary care. Br J Gen Pract 2010; DOI: 10.3399/bjgp10X483175.

2. Richards MA. The National Awareness and Early Diagnosis Initiative in England: assembling the evidence. Br J Cancer 2009; 101: s1-s4.

3. Richards MA. The size of the prize for earlier cancer diagnosis of cancer in England. Br J Cancer 2009; 101: S125-S129.

4. Abdel-Rahman M, Stockton D, Rachet D, et al. What if cancer survival in Britain were the same as in Europe: how many deaths are avoidable? Br J Cancer 2009; 101: S115-S124.

5. Moller H, Linklater KM, Robinson D. A visual summary of the EUROCARE-4 results: a UK perspective. Br J Cancer 2009; 101: S110-S114.

6. Neal RD, Allgar VL, Ali N, et al. Stage, survival and delays in lung, colorectal, prostate and ovarian cancer: comparison between diagnostic routes. Br J Gen Pract 2007; 57: 212-219.

7. Jones R, Charlton J, Latinovic R, Gulliford MC. Alarm symptoms and identification of non-cancer diagnoses in primary care: cohort study. BMJ 2009; 339: b3094. DOI: $10.1136 / \mathrm{bmj} . \mathrm{b} 3094$ 
35. Hamilton W, Lancashire R, Sharp D, et al. The importance of anaemia in diagnosing colorectal cancer: a case-control study using electronic primary care records. Br J Cancer 2008; 98: 323-27.

36. Hamilton W, Peters TJ, Round A, Sharp D. What are the clinical feature of lung cancer before the diagnosis is made? A population based casecontrol study. Thorax 2005; 60:1059-1065.

37. Barrett J, Hamilton W. Pathways to the diagnosis of lung cancer in the UK: a cohort study. BMC Fam Pract 2008; 9: 31.

38. Jones R, Latinovic R, Charlton J, Gulliford MC. Alarm symptoms in early diagnosis of cancer in primary care: cohort study using General Practice Research Database. BMJ 2007: bmj.39171.637106.AE

39. Hamilton W. MD thesis: Towards earlier diagnosis of cancer in primary care: a population-based case-control study of colorectal, lung and prostate cancer. Bristol, 2005

40. Ford AC, Veldhuyzen van Zanten SJO, Rodgers CC, et al. Diagnostic utility of alarm features for colorectal cancer: systematic review and meta-analysis. Gut. doi:10.1136/gut.2008.1597

41. Fijten G, Starmans R, Muris J, et al. Predictive value of signs and symptoms for colorectal cancer in patients with rectal bleeding in general practice. Fam Pract 1995; 12: 279-286.

42. Lawrenson R, Logle J, Marks C. Risk of colorectal cancer in general practice patients presenting with rectal bleeding, change in bowel habit or anaemia. Eur J Cancer Care 2006; 15: 267-271.

43. Wauters H, Van Casteren V, Buntinx F. Rectal bleeding and colorectal cancer in general practice: diagnostic study. BMJ 2000; 321: 998-999.

44. Du Toit J, Hamilton W, Barraclough K. Risk in primary care of colorectal cancer from new onset rectal bleeding: 10 year prospective study. BMJ 2006; 333: 69-70.

45. Ellis BG, Thompson M. Factors identifying higher risk rectal bleeding in general practice. Br J Gen Pract 2005; 55: 949-55.

46. Stapley S, Peters TJ, Sharp D, Hamilton W. The mortality of colorectal cancer in relation to the initial symptom and to the duration of symptoms: a cohort study in primary care. Br J Cancer 2006; 95: 1321-25.

47. Somasekar A, James L, Stephenson B, et al. The value of auditing negative lower GI investigations preceding a final diagnosis of colorectal cancer. Colorectal Disease 2009;11: 740-44.

48. Johnson CD, Chen M-H, Toledano AY, et al. Accuracy of CT Colonography for Detection of Large Adenomas and Cancers. N Engl J Med 2008; 359:1207-1217.

49. Ward DG, Suggett N, Cheng Y, et al. Identification of serum biomarker for colon cancer by proteomic analysis. Br J Cancer 2006; 94:1898-905.

50. Hurst NG, Stocken DD, Wilson S, et al. Elevated serum matrix metalloproteinase 9 (MMP-9) concentration predicts the presence of colorectal neoplasia in symptomatic patients. Br J Cancer 2007; 97: 971-977.

51. Loktionov A, Bandaletova T, Llewelyn A, et al. Colorectal cancer detection by measuring DNA from exfoliated colonocytes obtained by direct contact with rectal mucosa. Int J Oncology 2009; 34: 301-311.

52. Khan N, NCRI Colorectal Clinical Studies Group. Implementation of a diagnostic tool for symptomatic colorectal cancer in primary care: a feasibility study. Prim Health Care Res Dev 2009; 10: 54-64. doi:10.1017/S1463423608000996.
53. Soin G, Armitage J, McKay J, et al. Colorectal symptoms in the community - a ticking time bomb? Gut 2004; 53(suppl 3):Abstract 069.

54. Schroder FH, Hugosson J, Roobol MJ, et al. Screening and prostatecancer mortality in a randomized European study. N Engl J Med 2009; 360:1320-1328.

55. Andriole GL, Crawford ED, Grubb RL, et al. Mortality results from a randomized prostate-cancer screening trial. N Engl J Med 2009; 360:1310-1319.

56. Walsh PC. Surgery and the reduction of mortality from prostate cancer. N Engl J Med 2002; 347: 839-840.

57. Syrigos K. Prostate cancer. Oxford: Oxford University Press, 2001.

58. Hamilton W, Sharp D, Peters TJ, Round A. Clinical features of prostate cancer before diagnosis: a population-based case-control study. $\mathrm{Br} J \mathrm{Gen}$ Pract 2006; 56: 756-782.

59. Hamilton W, Sharp D. Symptomatic diagnosis of prostate cancer in primary care: a structured review. Br J Gen Pract 2004; 54: 617-621.

60. Issa MM, Zasada W, Ward K, et al. The value of digital rectal examination as a predictor of prostate cancer diagnosis among United States Veterans referred for prostate biopsy. Cancer Detect Prev 2006; 30 :269-275.

61. Gann $\mathrm{PH}$, Hennekens $\mathrm{CH}$, Stampfer MJ. A prospective evaluation of plasma prostate-specific antigen for detection of prostatic cancer. JAMA 1995; 273: 289-294.

62. Carter HB. Prostate cancers in men with low PSA levels - must we find them? N Engl J Med 2004; 350: 2292-2294.

63. Djavan B, Zlotta A, Kratzik C, et al. PSA, PSA density, PSA density of transition zone, free/total PSA ratio, and PSA velocity for early detection of prostate cancer in men with serum PSA 2.5 to $4.0 \mathrm{ng} / \mathrm{mL}$. Urology 1999; 54: 517-22.

64. Bankhead C, Collins C, Stokes-Lampard H, et al. Identifying symptoms of ovarian cancer: a qualitative and quantitative study. BJOG 2008; 115 : 1008-1014.

65. Tate A, Martin A, Murray-Thomas T, et al. Determining the date of diagnosis - is it a simple matter? The impact of different approaches to dating diagnosis on estimates of delayed care for ovarian cancer in UK primary care. BMC Medical Research Methodology 2009; 9(1): 42.

66. Bankhead C, Kehoe S, Austoker J. Symptoms associated with diagnosis of ovarian cancer: a systematic review. BJOG 2005; 112: 857-865.

67. Friedman GD, Skilling JS, Udaltsova NV, Smith LH. Early symptoms of ovarian cancer: a case-control study without recall bias. Fam Pract 2005 22: $548-53$.

68. Goff B, Mandel L, Melancon C, Muntz H. Frequency of symptoms of ovarian cancer in women presenting to primary care clinics. JAMA 2004; 291: 2705-2712.

69. Hamilton W, Peters TJ, Bankhead C, Sharp D. Risk of ovarian cancer in women with symptoms in primary care: population based case-contro study. BMJ 2009; 339(aug25_2): b2719. doi: 10.1136/bmj.b2719.

70. Hamilton W, Round A, Sharp D. Ovarian cancer. Not a silent killer.. BMJ 2009; 339(jul06_2): b2719-.

71. Rufford BD, Jacobs IJ, Menon U. Feasibility of screening for ovarian cancer using symptoms as selection criteria. BJOG 2007; 114: 59-64.

72. Sturgeon CM, Lai LC, Duffy MJ. Serum tumour markers: how to order and interpret them. BMJ 2009; 339(sep22_1): b3527-.

73. Menon U, Gentry-Maharaj A, Hallett R, et al. Sensitivity and specificity of multimodal and ultrasound screening for ovarian cancer, and stage distribution of detected cancers: results of the prevalence screen of the UK Collaborative Trial of Ovarian Cancer Screening (UKCTOCS) Lancet Oncol 2009; 10: 327-340.

74. Allhiser JN, McKnight TA, Shank JC. Lymphadenopathy in a family practice. J Fam Pract 1981;12: 27-32.

75. Williamson HA, Jr. Lymphadenopathy in a family practice: a descriptive study of 249 cases. J Fam Pract 1985; 20: 449-452.

76. Fijten GH, Blijham GH. Unexplained lymphadenopathy in family practice. An evaluation of the probability of malignant causes and the effectiveness of physicians' workup. J Fam Pract 1988; 27: 373-376.

77. Hamilton W, Kernick D. Clinical features of primary brain tumours: a case-control study using electronic primary care records. $\mathrm{Br} \mathrm{J} \mathrm{Gen} \mathrm{Pract}$ 2007; 57: 695-699.

78. Danaei G, Vander Hoorn S, Lopez AD, et al. Causes of cancer in the world: comparative risk assessment of nine behavioural and environmental risk factors. Lancet 2005; 366: 1784-1793.

79. Hampton J. Guidelines for the obedience of fools and the guidance of wise men? Clin Med 2003; 3: 279-284. 